\title{
Detector R\&D
}

\section{Bortoletto*}

Department of Physics

University of Oxford

E-mail: daniela.bortoletto@cern.ch

The Detector R\&D parallel session at ICHEP 2018 was especially rich. It included over 90 contributions outlining the amazing performance of current detectors and the outstanding progress made by our community towards future experiments at new facilities that are in various stages of planning. In this paper a selected summary of this rich program is presented, focusing on the $\mathrm{R} \& \mathrm{D}$ for the future of our field.

The 39th International Conference on High Energy Physics (ICHEP2018) 4-11 July, 2018 Seoul, Korea

* Speaker. 


\section{The physics Landscape}

Despite the amazing success of the Standard Model many significant questions remains unanswered. What is the nature of the Higgs boson? What can we learn from discovering that neutrinos have mass? Can the known forces be further unified? The particles of the Standard Model make up only $5 \%$ of the universe, what is the other $95 \%$ ? Why is the universe almost all matter and no antimatter? Future facilities are planned to address these fundamental questions. Experiments at these facilities are ambitious, large-scale projects that require novel and challenging experimental techniques. Most of the significant ongoing R\&D to address these challenges was presented at this conference. Here I present a selected summary.

\section{The plan for the LHC upgrades}

The Large Hadron Collider (LHC) is the most powerful particle accelerator built to date. The scientific programme of the LHC started almost 10 years ago and will continue over the next 20 years. In Run 2 (2015-2017), the machine has operated at a peak luminosity of up to $2 \times 10^{34}$ $\mathrm{cm}^{-2} \mathrm{~s}^{-1}$ delivering $\approx 150 \mathrm{fb}^{-1}$ to both ATLAS and CMS at a center of mass energy of $13 \mathrm{TeV}$. The machine will be a upgraded in several steps during two long shutdowns lasting between two and three years. The various steps to reach the high-luminosity phase (HL-LHC) are indicated in Figure 1. During Long Shutdown 2 (LS2), that starts in 2019, the LHC injectors will be upgraded and the dipole magnets will be trained to reach $7 \mathrm{TeV}$ allowing operation at $14 \mathrm{TeV}$. Long Shutdown 3 (LS3) starting at the end of 2023 will include major performance upgrades of the accelerator for the HL-LHC. In the HL-LHC stage the machine will operate at a peak luminosity between 5 and $7 \times 10^{34} \mathrm{~cm}^{-2} \mathrm{~s}^{-1}$ and will deliver between 3000 and $4000 \mathrm{fb}^{-1}$. This increase in performance brings an average number of interactions per crossing (pile up) between 140 and 200 and requires the replacement of several major detector components in both ATLAS and CMS. The detectors, including the main tracking systems, must be rebuilt to deal with the extreme radiation levels and large numbers of interactions per beam crossing. Technical Design reports $[1,2]$ have been completed for many of these upgrades, and some projects will start the pre-production phase soon,

LHCb runs at a lower luminosity than ATLAS and CMS, to avoid excessive pile-up since it is designed to perform precision studies of heavy flavours quarks. The total luminosity collected by LHCb in Run 2 is nearly $6 \mathrm{fb}^{-1}$, twice the Run 1 sample of $3 \mathrm{fb}^{-1}$. This corresponds to an enormous sample of the order of $2 \times 10^{12} \mathrm{~B}$ decays. The instantaneous luminosity will increase by a factor of five from $\mathrm{L}=4 \times 10^{32} \mathrm{~cm}^{-2} \mathrm{~s}^{-1}$ in Run 1 and Run 2 to $\mathrm{L}=2 \times 10^{33} \mathrm{~cm}^{-2} \mathrm{~s}^{-1}$ in Run 3. To cope with this change in luminosity LHCb Upgrade I [3] will be installed during LS2 to unable data taking in 2021. During LS3 a consolidation of the LHCb Upgrade I detector, denoted ads Upgrade Ib, will occur. LHCb Upgrade II [4] will be installed during LS4, with operations beginning in LHC Run 5, which is scheduled to start in 2031. The Upgrade II experiment will operate at instantaneous luminosities of up to $2 \times 10^{34} \mathrm{~cm}^{-2} \mathrm{~s}^{-1}$, an order of magnitude above Upgrade I. LHCb will accumulate a data sample corresponding to a minimum of $300 \mathrm{fb}^{-1}$. The planned increase in luminosity for LHCb is illustrated in Figure 2. 


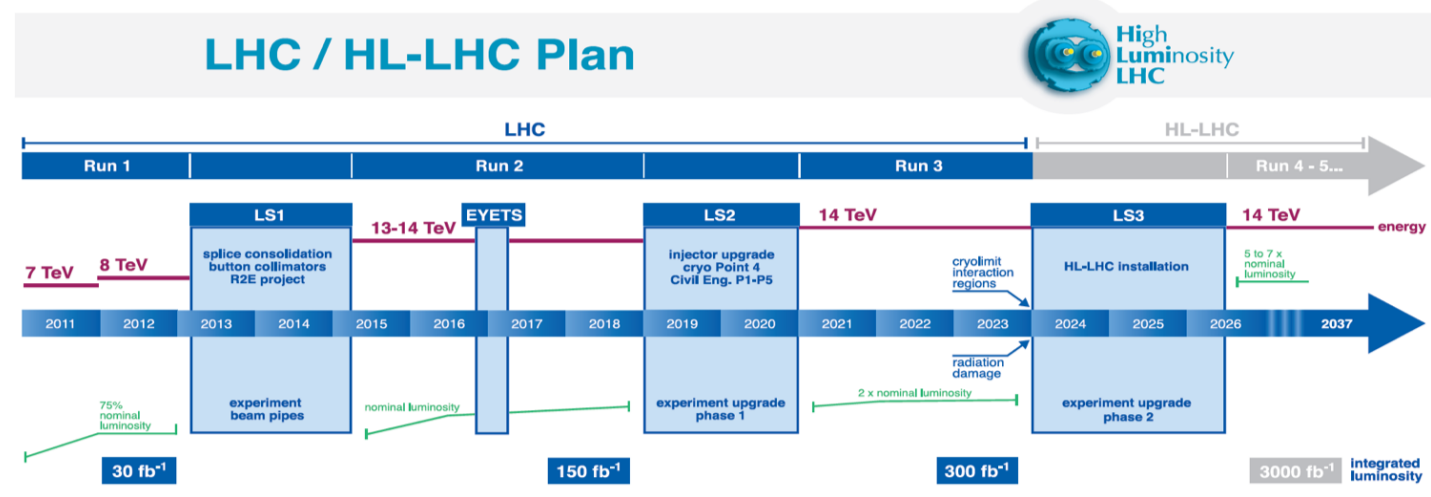

Figure 1: CERN technical plan for operations of the LHC over the next decade.

\section{The LHCb Upgrade I}

One of the major changes during LHCb Upgrade I is the installation of a completely new DAQ system[5] without any low level hardware trigger, providing higher trigger yield at the luminosity foreseen after LS2. The current readout and triggering limits the physics reach of the experiment since the trigger yield of many hadronic channels saturates already at run 2 instantaneous luminosities. In order to overcome this problem the LHCb trigger will be upgraded during LS2. In this upgrade the L0 hardware-based trigger will be removed, and events will be selected by a softwarebased HLT which will perform full event reconstruction at an event rate of $30 \mathrm{MHz}$. In order to transport the full data rate, custom FPGA readout boards (PCIe40) and state of the art off-the-shelf network technologies will be used. The readout signals will be sent to the surface by $300 \mathrm{~m}$ long optical links to an event builder farm. Offline precision particle identification and track quality information will be exploited to reduce the rate down to $20-100 \mathrm{KHz}$. The new trigger strategy will increase the triggering efficiency on the hadronic channels by a factor 2 to 4 with respect to the current performance. This corresponds to an increase by a factor of 10 to 20 in the yield of hadronic events. To implement this trigger the readout of many LHCb subdetectors were upgraded so that they could all be read out at the at full collision rate of $40 \mathrm{MHz}$.

The Vertex Locator (VELO) is an essential part of the LHCb tracker providing precise measurement of primary vertices and displaced vertices of short living particles. The current detector is built using silicon strip sensors surrounding the interaction point. The VELO upgrade [3] includes 26 tracking layers based on $55 \times 55 \mu \mathrm{m}^{2}$ pixel sensors that will ensure excellent hit resolution and simpler track reconstruction. VELO will operate in a secondary vacuum separated from the primary LHC vacuum by a thin metal foil, screening VELO from the RF radiation of the beam 

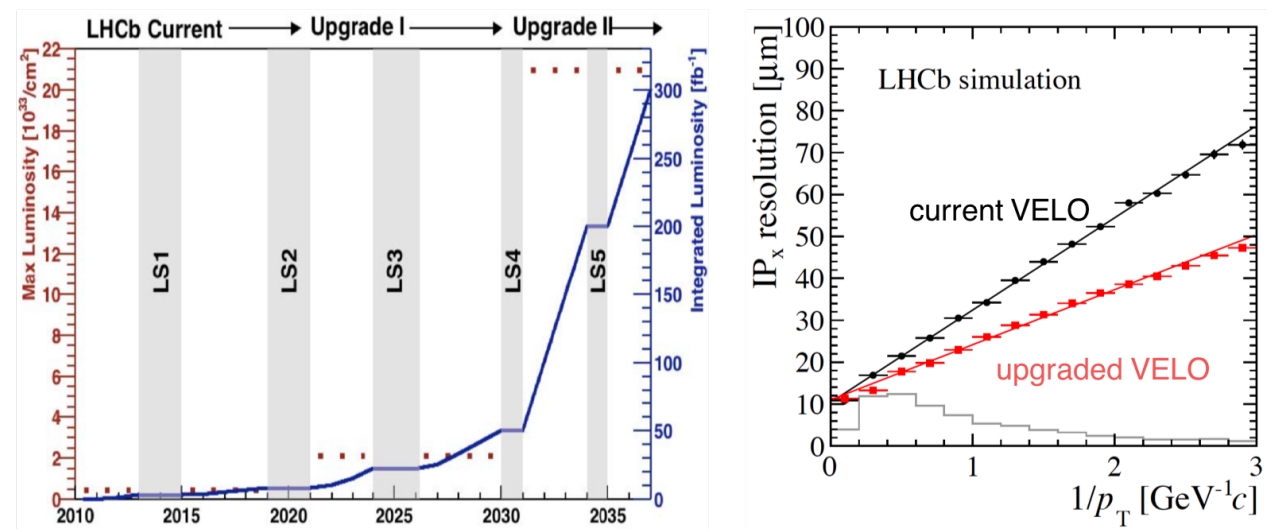

Figure 2: Evolution of the maximum Instantaneous Luminosity and integrated luminosity for LHCb (left). The improvement in impact parameter resolution brought by the VELO upgrade (right).

and preventing the VELO components from contaminating the LHC vacuum. The foil contributes significantly to the total material budget and the aim is to reduce its thickness to $<300 \mu \mathrm{m}$. The upgraded VELO will be closer to the beam since its closed position will reach $5.1 \mathrm{~mm}$ instead than the current $8.2 \mathrm{~mm}$. The detector material before the intersection of the first tracking layer is decreased from $4.6 \%$ to $1.7 \%$ radiation lengths. These changes improve the impact parameter resolution by a factor of about $40 \%$ (Figure 2), increase the VELO tracking efficiency especially for low momentum tracks, and provide a better decay time resolution. In order to reduce the radiation damage to the sensors, the detector layers will be opened while not taking data and closed when stable beam condition is reached. The sensors will be cooled at the temperature of $-20 \mathrm{C}$ exploiting an innovative microchannel $\mathrm{CO}_{2}$ cooling technology sketched in Figure 3. The upgraded VELO will use a new VeloPix ASIC that can be read out at $40 \mathrm{MHz}$ and a bandwidth of up to $20.4 \mathrm{~Gb} / \mathrm{s}$.

\section{ATLAS and CMS upgrades}

\subsection{New Tracking Systems for the HL-LHC}

Both CMS and ATLAS will be replacing their trackers in LS3 with detectors designed to withstand the HL-LHC planned luminosity. The layout of the new detectors is shown in figure 4. The two collaborations have made some similar choices in the development of the new detectors. For examples both detectors will include an innermost part equipped with pixels sensors sourranded by strip layers. The inner barrel of the CMS tracker and the ATLAS pixel detector have adopted novel tilted geometry for better tracking and reduced material. Cooling will be provided by similar systems using two-phase $\mathrm{CO}_{2}$. Power will be delivered using radiation hard DC-DC conversion or serial powering technology. In order to cope with the HL-LHC radiation environment both CMS and ATLAS are planning to have the innermost pixel layers to be replaceable. The silicon 

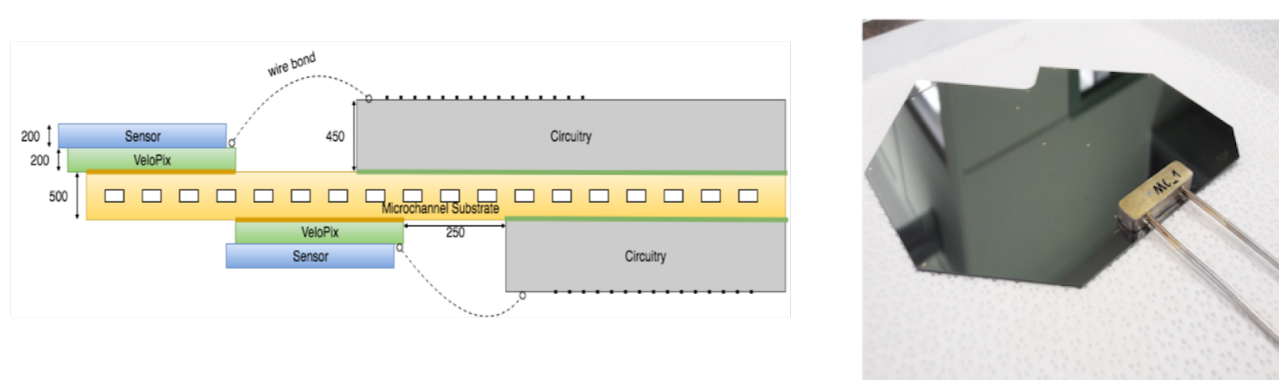

Figure 3: A sketch of the VELO microchannel cooling concept (left) and a photograph of its implementation (right).

strip sensors are fabricated using the p-in-n technology allowing the collection of electrons which have a higher drift velocity and are less subject to trapping after irradiation. To cope with the high radiation levels which must be sustained by the pixel detectors, a readout chip using $65 \mathrm{~nm}$ CMOS technology and an architecture where a group of channels shares digital electronics has been developed by the RD53 collaboration for both CMS and ATLAS.
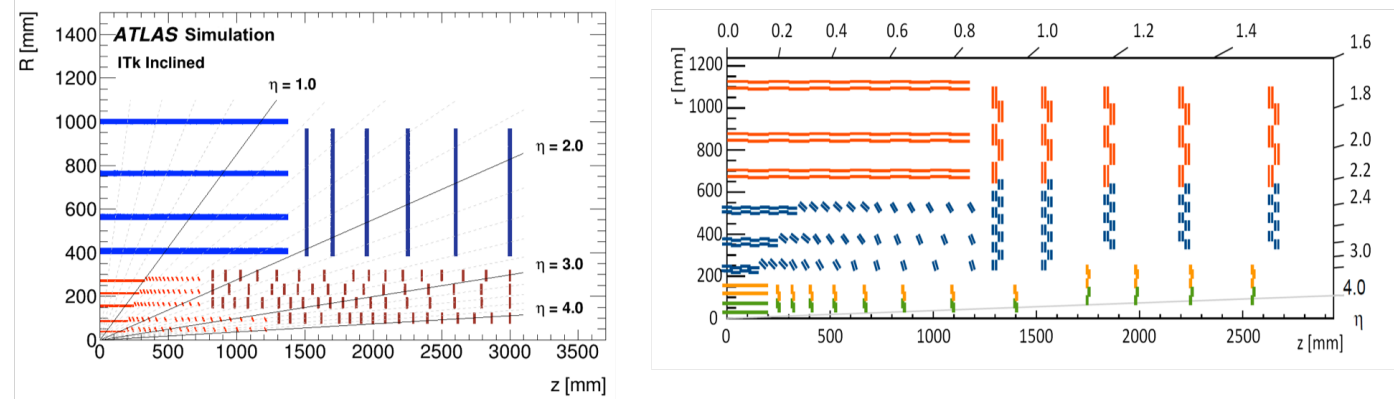

Figure 4: The layout of the ATLAS ITk (left) and the CMS tracker (right) for the HL-LHC

The new ATLAS Strip tracker system, the ITk Strip detector[1], has roughly 60 million channels and a silicon area of $165 \mathrm{~m}^{2}$, which is 2.5 times more than the Semiconductor Tracker (SCT). The large area will be covered by 17,888 modules consisting of one silicon sensor and low-mass printed circuit boards, called hybrids, directly glued onto it. Modules are glued on local support structures. They consist of two polyamide bus tapes co-cured to carbon fibre face-sheets, which sandwich a low-density carbon-fibre honeycomb core. Titanium cooling pipes surrounded by a high conductivity thermal foam, are embedded inside the structure. This design leads to thin structures called staves in the barrel and petals in the forward region that achieve lower radiation length 
per layer than the SCT. Stave and petal prototypes are undergoing various tests, ranging from optical metrology, mechanical bending and stress, and thermal cycling. The ATLAS strip detector covering $\eta<2.7$ is complemented by a large five layer high precision Pixel Detector which extend the coverage to $\eta<4$, as shown in Figure 4. The combined system, denoted as ITk provides 9 space points in the $\eta<4$ volume. The new detector has been designed with less inactive material in the tracking volume and will deliver excellent tracking and vertexing even with an average pileup of 200 . The pixel cell size will be reduced to $50 \times 50 \mu \mathrm{m}^{2}$ or $25 \times 100 \mu \mathrm{m}^{2}$ to improve intrinsic spatial resolution and two track separation. The choice between these two geometries will be based on physics reach and technical feasibility. The inner two pixel layers are separated from the outer layers by an Inner Support Tube facilitating their replacement.

The CMS pixel detector [2] comprises four barrel layers and eleven forward pixel disks while the CMS outer tracker has six layers. The Outer Tracker starting at a radius $r>20 \mathrm{~cm}$ contains two types of modules, which are made of a stack of two silicon sensors with a gap ranging from 1.6 to $4.0 \mathrm{~mm}$. These modules are designed to reduce the amount of data being transmitted by applying a cut on the transverse momentum of charged particles, exploiting the high magnetic field of CMS, as illustrated in Figure 5. A programmable search window allows to select high momentum particles removing $99 \%$ of the particles with $p_{T}<2 \mathrm{GeV} / \mathrm{c}$. The accepted short track segments including the position and bend information are transferred to the back-end via high-speed optical links and provide fitted track parameters to the L1-trigger system within $4 \mu \mathrm{s}$. This massive computation task is performed on Track Finding Processors based on FPGAs. The feasibility of track finding within the given time window of $4 \mu$ s with transverse momentum resolution of about $2 \%$, longitudinal vertex position resolution of about $1 \mathrm{~mm}$, yielding a significant rate reduction of $1 / 100$ was demonstrated in hardware by three different approaches. The CMS pixel detector will have an active surface of approximately $4.9 \mathrm{~m}^{2}$ and consist of 4352 modules with $1.947 \times 10^{9}$ pixels.
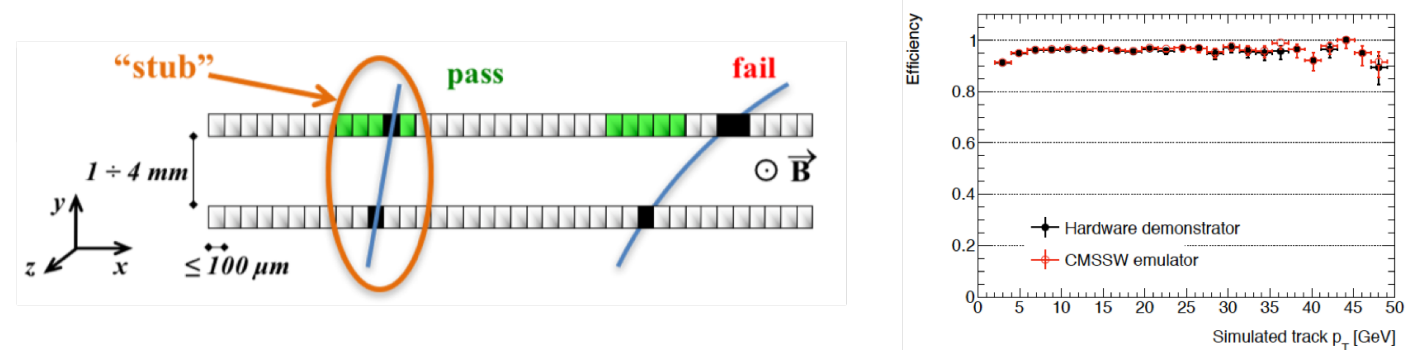

Figure 5: A sketch of the CMS track-triggering concept (left). Track reconstruction efficiency, measured in both hardware and emulation, for tracks originating from the primary interaction in $t \bar{t}$ events with $200 \mathrm{PU}$, as a function of $p_{T}$

\subsection{New Timing Detectors for the HL-LHC}

Particle reconstruction with a pileup of 240 is extremely challenging therefore both ATLAS and CMS are planning to use precision timing in the HL-LHC era. Time tagging of charged particles produced in LHC collisions provides further discrimination of interaction vertices in the same $25 \mathrm{~ns}$ bunch crossing beyond what can be achieved by using only spatial tracking algorithms, as it is currently done. According to preliminary simulation studies, a timing resolution of about $30 \mathrm{ps}$ 
allowing to distinguish between collisions occurring very close in space but well-separated in time, as illustrated in Figure 6.

CMS plans to install a dedicated Minimum Ionizing Particle timing detector (MTD)[6] which will provide timing information with about $30 \mathrm{ps}$ resolution for charged tracks. A single layer of LYSO crystals read out by silicon photomultipliers (SiPMs) will be used in the barrel region while in the end cap region silicon Low Gain Avalanche Detectors (LGADs) will provide timing information up to $|\eta|<3.0$. Radiation tolerance, integration, services, schedule constraints, and cost lead to the selection of different technologies for the barrel and for the end caps. According to preliminary simulation studies, the instances of vertex merging are reduced on average from $15 \%$ in space to $1 \%$ in space-time. The MTD improves the performance of b-jet identification, which relies on vertex reconstruction, and the efficiency of final state observables relevant for the identification of Higgs boson production and decays such as Higgs boson decays to four leptons and di-Higgs boson events. The removal of pileup tracks from the isolation cones improves the identification efficiency for isolated leptons and photons. Furthermore the ability to reconstruct the time of displaced vertices will provide enhanced capabilities in the search of long-lived particles.
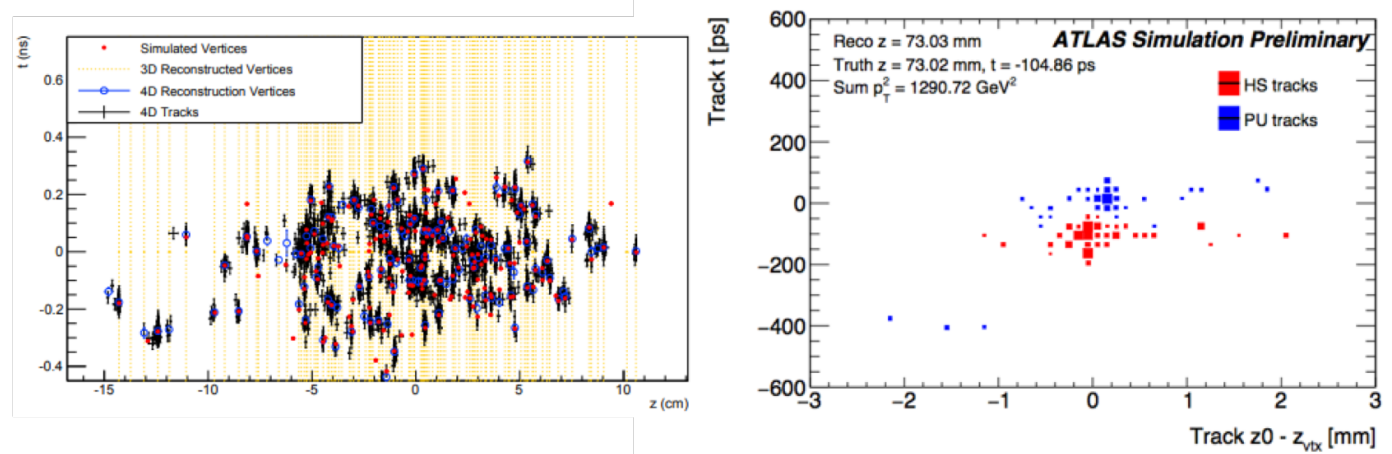

Figure 6: The importance of timing detectors to distinguish interaction from hard scattering and pileup with an average pileut of 200. The CMS (left) simulation shows that vertices merged in 3D (yellow lines) are separated in 4D using timing. The ATLAS (right) simulation of a VBF-produced Higgs boson decaying invisibly $(H \rightarrow Z(v v) Z(v v))$ are superimposed along $\mathrm{z}$ while the interactions are well separated in time.

The ATLAS High-Granularity Timing Detector (HGTD) will use LGADs providing a time resolution of $30 \mathrm{ps} /$ track in the region of $2.4<|\eta|<4.0$. This new detector will significantly improve the track-to-vertex association in the forward region, compensating for the reduced longitudinal impact parameter resolution of tracks reconstructed by the ITk tracker at large $\eta$. The HGTD improves the rejection of pileup jets by $\approx$ a factor of 2 (for a hard-scatter jet efficiency of $98 \%$ ) and increases the lepton isolation efficiency by $14 \%$. Furthermore the light- jet rejection at a b-jet efficiency of $70 \%$ improves by a factor of 1.5 . The better object reconstruction performance translates into sensitivity gains and enhances the reach of the HL-LHC physics program in the forward region such as studies of VBF-produced Higgs bosons decaying to $H \rightarrow W W$. The HGTD also provides unique capabilities to measure the online and offline luminosity with high accuracy. The HGTD consists of two $75 \mathrm{~mm}$ disks covering the radial range $11<R<100 \mathrm{~cm}$ installed between the ITK and the endcap-forward calorimeters at a distance of $+3.5 \mathrm{~m}$ and $-3.5 \mathrm{~m}$ from the interaction point. In order to keep a $30 \mathrm{ps} /$ track during its full life time, two double sided layers 
of LGAD will overlap in the inner ring $(3.2<|\eta|<4.0)$, which is exposed to highest irradiation doses, to achieve 3 hits per track while in the external ring covering the region $2.4<|\eta|<3.2$ only 2 hits/track will be available. The inner ring will be replaced after a fluence of $3.7 \times 10^{15} \mathrm{n}_{e q} / \mathrm{cm}^{2}$ and a TID of 4.0 MGy. The sensor, of size of $2 \times 4 \mathrm{~cm}^{2}$, will contain macro-pixel of $1.3 \times 1.3$ $\mathrm{mm}^{2}$ granularity. The signals will then be transmitted at various speeds depending on the radial position of the ASIC through optical links to the ATLAS USA15 electronics cavern. A total of 6.3 $\mathrm{m}^{2}$ modules are needed to equip the full detector to be readout by 3.54 Million channels. A major R\&D activity is the ASIC-ALTIROC development to readout the LGAD sensors which is driven by the required $30 \mathrm{ps}$ time resolution per mip after irradiation.

\section{Future Colliders}

The particle physics community is planning future Colliders beyond the HL-LHC. CERN is exploring several circular collider options, FCC (ee, hh, eh), and the CLIC linear $\mathrm{e}^{+} \mathrm{e}^{-}$accelerator. Other developments are discussed in China (CepC, CppC) and Japan (ILC). These high energy frontier projects push to very challenging values the performance of the required detectors[8], as illustrated by these examples.

The detector requirements at a future circular hadron collider, such as FCC-hh or CppC which plan to reach a centre of mass energy of about $100 \mathrm{TeV}$, are truly challenging. The planned operation scenarios aim at a peak luminosity of $30 \times 10^{34} \mathrm{~cm}^{-2} \mathrm{~s}^{-1}$ and an integrated luminosity of 30 $\mathrm{ab}^{-1}$. Therefore the detectors will have to stand radiation levels that are about a factor of 10-100 worst than the HL-LHC, a pile-up of up to 1000, and data rates of TB/s. In order to limit the detector size, a very large and strong solenoid magnet, complemented by smaller magnets in the forward areas will be necessary. The determination of the timing of tracks will be critical for pile-up mitigation leading to a timing precision requirement of 5-10 ps. Software to handle the expected data rates is not available today. The expected radiation levels at the innermost layers call for major improvements in the tracking sensor technology. Progress is also needed in the electromagnetic and hadronic calorimetry.

Future lepton colliders, such as CLIC, ILC, FCC-ee, anc CepC will require a radically different detector optimization. Ultra precision precision tracking at the level of a few $\mu \mathrm{m}$ must be achieved to distinguish Higgs boson decays to charm from those to bottom quarks. Therefore the innermost part of these systems must have silicon pixel detectors with a very small cell size and a total material between 0.2 and $0.3 \% \mathrm{X}_{0} /$ layer. Current candidates for these sensors are monolithic devices mounted on ultra light structures. New materials with excellent thermal conductivity such as carbon nanotubes and graphene should be considered. Highly granular calorimetry to allow particle-flow reconstruction or dual read calorimetry will be needed to separate $Z$ from $W$ decays for precision physics.

\section{Neutrino Experiments}

Many open questions will be addressed by a new generation of neutrino detectors[9] such as the discovery and study of leptonic CP violation, the determination of the neutrino mass hierarchy, the measurements of the oscillation parameters, and the discovery of their true nature, Dirac or 
Majorana. Novel massive neutrino detectors are under construction in the US, Japan, and China. There was a special section on neutrinos and I will limit my comments to the Jiangmen Underground Neutrino Observatory (JUNO).

JUNO is a large liquid scintillator detector located $53 \mathrm{~km}$ from a reactor that aims at determining the neutrino mass hierarchy. The detector will be located 1800 meters of water equivalent (m.w.e.) underground and consists of a 20 kiloton LAB liquid scintillator contained in a $35.4 \mathrm{~m}$ diameter acrylic sphere. The attenuation length of the liquid scintillator is longer than $20 \mathrm{~m}$ at 430 $\mathrm{nm}$. The vessel is instrumented with more than 17000 20-inch and about 34000 3-inch PMTs that will be mounted in gaps between large phototubes, a shown in in Figure 7. This configuration will ensure a $77 \%$ coverage. An energy resolution of $3 \% \sqrt{E(\mathrm{MeV})}$ and an absolute energy scale of $1 \%$ are required to measure the mass hierarchy. The antineutrino event will be identified observing the inverse beta decay and the delayed signal of $2.2 \mathrm{MeV}$ from the neutron captured by hydrogen within 200 s. Two types of 20" PMTs have been developed to achieve a high light yield of 1200 p.e./MeV and a quantum efficiency of $27 \%$. One type of PMT is fabricated by Hamamatsu. Researchers at IHEP, Beijing have developed a new PMT concept where a small Microchannel Plate (MCP) replaces the bulky Dynode chain used in traditional large PMTs. The PMTs were then industrialized with NNVT. Both PMT types have reached the performance needed to obtain the required energy resolution (Figure 7). JUNO plans to start data taking in 2020.
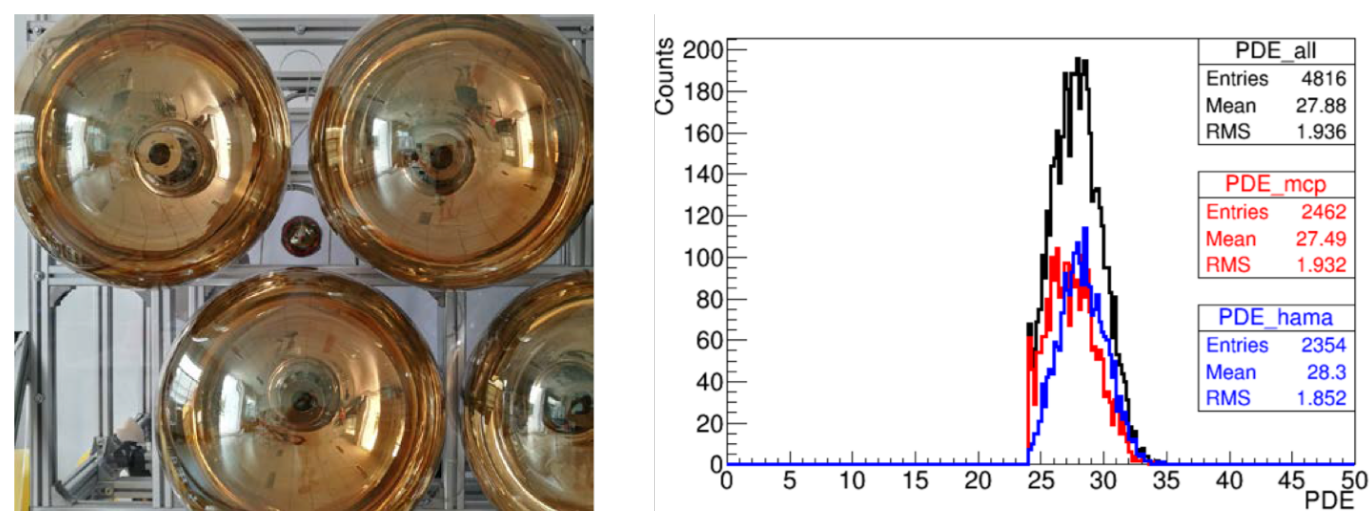

Figure 7: The configuration of the PMTs in JUNO (left). A comparison of the perfromance of the PMTs from Hamamtsu and NNVT

\section{Important Technology Developments}

Major advances in the development of an array of technologies have taken place recently. Some of these developments are described below.

\subsection{Depleted Monolithic Pixel Sensors}

The use of commercial CMOS technologies to produce a monolithic device with the pixel 
sensor and its electronics on the same wafer was proposed[10] already in the early 1990s. Since then monolithic active pixel sensor (MAPS) detectors have been operating in several experiments in heavy ion physics[11]. The maximum charge deposited in these devices is about 4,000 $\mathrm{e}^{-}$ because the epi-layer, which hosts the CMOS circuitry, is only about $15 \mu \mathrm{m}$ thick. Since the epi-material is often low resistivity and CMOS technologies operate at low voltages, the epi-layer usually is depleted only locally around the charge collection node. Therefore, the charge deposited by a charged particle traversing the device is mostly collected by diffusion rather than by drift, making the technology not suitable for the LHC or the HL-LHC. Recently High Voltage CMOS technologies and high resistivity substrates $(>100 \Omega \mathrm{cm}$ ) have become available as process add-ons or modifications, making these detectors more interesting for the outer layer of the HL-LHC pixel system. Multiple nested wells can be used to isolate transistors and shield deep well potentials in order to optimize charge collection. The introduction of a backside biasing contact can also improve field shaping potential of the device.
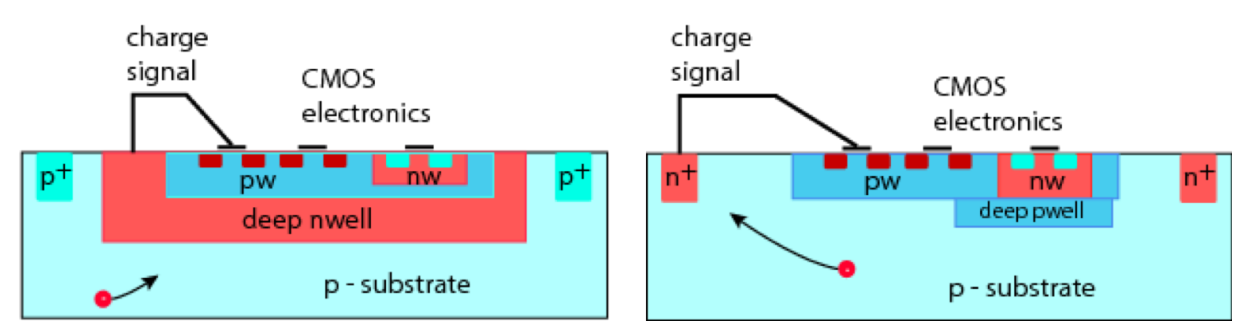

Figure 8: The large fill-factor (left) and the small fill-factor (right) architecture adopted for Depleted CMOS detectors.

The ATLAS collaboration initiated an effort for evaluate the potential of this technology looking at two options, which are shown in Figure 8. In the large fill-factor configuration the entire CMOS electronics is enclosed in the deep charge collecting n-well while in the small fill-factor option the charge is collected at a small n-well located outside the electronics area. The large fill-factor offers good charge collection properties over the entire pixel area but is affected by a larger inter-well capacitance $(\approx 100 \mathrm{fF})$ and therefore it yields larger noise and slower timing than the small-fill approach. Small prototypes were fabricated with ams, LFOUNDRY, and Tower Jazz covering the large fill-factor with the first two vendors and the small fill-factor with the last one. The initial results have been extremely promising.

The HV technology (called HVCMOS) with $350 \mathrm{~nm}$ and later with $180 \mathrm{~nm}$ feature sizes (AMS H35 and H18) showed an achievable depletion depth of around 15-30 $\mu \mathrm{m}$ for low resistivity substrates around $10 \Omega \mathrm{cm}$. These devices have been tested to TIDs well above $100 \mathrm{Mrad}$ and fluences up to $5 \times 10^{15} \mathrm{n}_{e q} \mathrm{~cm}^{2}$. Test beam characterization of prototype matrices coupled to the FE-I4 pixel chip via adhesive bonding yielded efficiencies of above $97 \%$ and in-time efficiencies comparable to those of FE-I4 hybrid pixel modules used in the same test beam. Prototype matrices produced by LFoundry LF15A on a high resistivity substrate wafers $(2-3 \mathrm{k} \Omega \mathrm{cm})$ were also tested up to fluences of $1 \times 10^{15} \mathrm{n}_{e q} \mathrm{~cm}^{2}$ and TIDs of $50 \mathrm{Mrad}$. The devices performed well in test beams but the in-time efficiency was below $91 \%$. The radiation hardness of the small fill-factor design, which was improved by modifying the TJ process and by replacing the epitaxial p-layer by a planar 
deep n-p junction, has also shown promising results and its performance after irradiation is under investigation.

\subsection{The LGAD technology}

The Ultra-Fast Silicon Detectors [12] used in the CMS and ATLADS upgrade are based on Low-Gain Avalanche Detectors (LGAD). The design of an LGAD consists of highly doped $\mathrm{n}^{++}$ and $\mathrm{p}^{++}$electrodes on the surface of a p-substrate. The doping profile is then modified by introducing an additional doping layer of $\mathrm{p}+$ material (Boron or Gallium) underneath the $\mathrm{n}^{++}$electrode to create a very high electric field of up to $300 \mathrm{kV} / \mathrm{cm}$, as shown in Figure 9. The main contributions to the time resolutions are due to jitter and time walk which can be minimized by achieving a fast signal rise, low intrinsic noise, and low noise amplification. Therefore, LGADs should be thin, in order to have a faster rising edge, but not too thin since a large value of the capacitance increases the capacitance and therefore the noise. Precise timing resolution also requires electrode geometries maintaining, as much as possible, uniform carriers' drift velocity and weighting fields. Thus, in order to preserve position resolution, the sensor thickness should be of about the same dimension as the pad size.
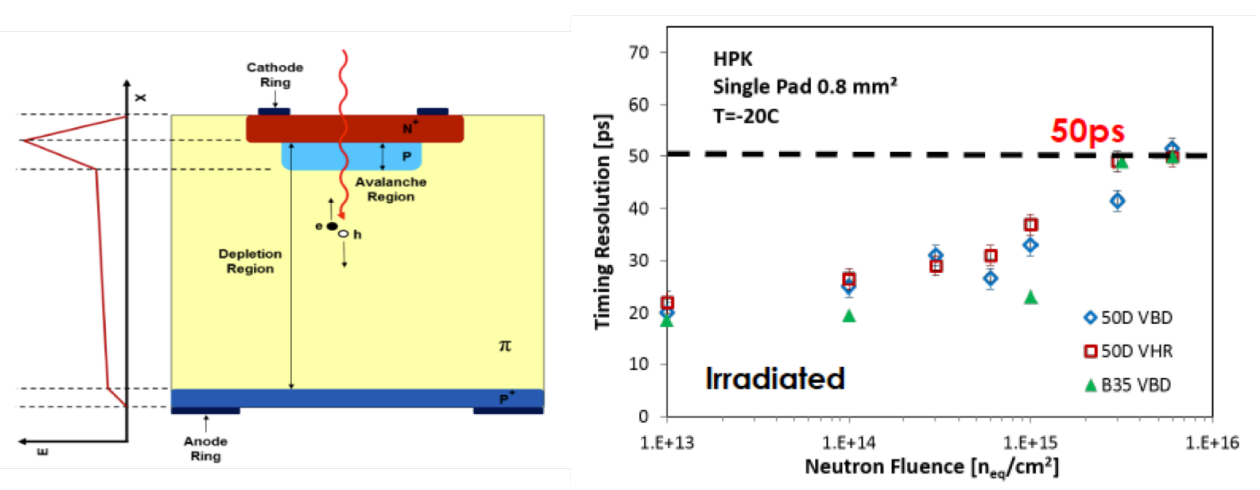

Figure 9: Sketch of the LGAD concet (left). Performance of LGAD sensors as a function of the dose(right).

The R\&D conducted for the timing layers of CMS and ATLAS has shown that $50 \mu \mathrm{m}$ thick LGAG can reach a timing resolution $\sigma(\mathrm{t})=34 \mathrm{ps}$ for a gain of about 20 and $\sigma(\mathrm{t})=20$ ps when averaging the response of three detectors. A major concern for the use of LGADs at the HL-LHC is their performance in a high radiation environment, as shown in (Figure 9). LGADs have shown sensitivity to radiation damage since the increase in leakage current due to radiation effects is multiplied by the gain value. Moreover, the junction providing the amplification gain requires pdoping concentrations of order of $10^{16} / \mathrm{cm}^{3}$ and radiation induced acceptor removal will reduce the gain. The increase in leakage current can be reduced by using thinner sensors and with operation at lower temperatures. R\&D is ongoing in RD50 and AIDA2020 to mitigate the reduction of gain due the changes in doping profile. The effort includes reducing the concentration of interstitials available for capturing Boron atoms by using carbon enriched wafers and by replacing Boron with Gallium to reduce interstitial capture that scales with atomic mass.

\subsection{Silicon photomultipliers}

Silicon Photomultipliers (SiPMs), are increasingly used in particle physics[13] as shown in 
this conference. The impact of SiPM is due to the fact that bring many advantages such as small size, a high photon detection efficiency, insensitivity to magnetic fields, high intrinsic gain, fast response, low operating voltages and a level of radiation hardness sufficient for many applications. Unfortunately, they suffer from a finite dynamic range, voltage-dependent noise, and a strong temperature dependence of the breakdown voltage.

SiPMs have a wide variety of applications from calorimetry to tracking. They will be used for the "tile-on-SiPM" technology adopted for the scintillator part of the phase II upgrade of the highly granular sampling calorimeter CMS end-cap calorimeter system[2]. The SiPMs, that are used to readout the electromagnetic calorimeter of $\mathrm{Mu} 2 \mathrm{e}$, are UV-extended sensors optimized to provide sensitivity to the $315 \mathrm{~nm}$ photons produced by the fast scintillation component of CsI crystals. Hamamatsu SiPM arrays with a silicon entrance window have demonstrated a time resolution of $110 \mathrm{ps}$ and an energy resolution of 7\%. For the MEG experiment liquid Xe calorimeter upgrade the key challenge is the development of SiPMs which are sensitive to liquid Xe scintillation light $(\lambda=175 \mathrm{~nm})$. To achieve this VUV sensitivity, several modifications with respect to standard SiPMs are implemented. The contact layer is thinned to improve the efficiency for the detection of short wavelengths. In addition, the protection layer of resin and the anti-reflective coating are removed and replaced by a VUV-transparent quartz window to improve the optical matching between the liquid $\mathrm{Xe}$ and the sensor surface. The devices use a metal quench resistor to achieve a smaller temperature coefficient. These modifications yield a photon detection efficiency of $\approx 20 \%$ for liquid Xe scintillation light which is similar to the performance of PMTs.

SiPMs are also used in many trackers. For example a SiPM-based fiber trackers will be installed in the upcoming shutdown for the upgrade of the LHCb experiment. A smaller system will be built for the Mu3e experiment, where a cylindrical three-layer scintillating fiber detector will be used for tracking and precision timing. New SiPMs are currently being developed for photon detection in the DUNE liquid argon TPCs. These devices include wavelength-shifting to match the $128 \mathrm{~nm}$ VUV scintillation light from the liquid argon to the spectral sensitivity of the photon sensors and provide photon collection over areas much larger than the active sensor area. For this application cryogenic operation is required.

After-pulsing and inter-pixel crosstalk have been considerably reduced by introducing of optical barriers between microcells. The operation at low temperatures on the order of 30 to $40 \mathrm{C}$ reduces significantly the dark rate and noise induced by radiation damage. This enables the use of SiPMs in areas of high fluence for LHC calorimeters. The improved performance and the reduction of price per unit area will continue to open more possibilities to the application of SiPMs. The market of SiPMs is evolving rapidly with several producers now available allowing the production not only of standard devices but also of specialized protection layers and entrance windows to enable VUV sensitivity.

\section{Conclusions}

The R\&D effort for the LHC upgrades and for the new generation of experiments at the intensity frontier have lead to amazing progress in detector technology. New challenges remain in front us and will have to be solved to meet the requirements set by future colliders. 


\section{Acknowledgment}

I would like to thank the organisers of ICHEP who have very successfully run a memorable conference. I would also like to thank the conveners and participants of the R\&D parallel session for their effort and for the excellent presentations covering many interesting topics.

\section{References}

[1] The ATLAS Collaboration, Technical Design Report for the ATLAS Inner Tracker Strip Detector, CERN-LHCC-2017-005. ATLAS-TDR-025, CERN, 2017; The ATLAS Collaboration, Technical Design Report for the ATLAS Inner Tracker Pixel Detector, CERN-LHCC-2017-021. ATLAS-TDR-030, CERN, 2018.

[2] The CMS Collaboration, The Phase-2 Upgrade of the CMS Tracker, CERN-LHCC-2017-009. CMS-TDR-013, CERN, 2017; The CMS Collaboration, The Phase-2 Upgrade of the CMS Endcap Calorimeter, CERN-LHCC-2017-023. CMS-TDR-019, CERN, 2017.

[3] The LHCb Collaboration, LHCb Trigger and Online Upgrade Technical Design Report, CERN-LHCC-2014-016, LHCB-TDR-016, 2016; The LHCb Collaboration, LHCb PID Upgrade Technical Design Report, CERN-LHCC-2013-022, LHCB-TDR-014, 2014; The LHCb Collaboration, LHCb VELO Upgrade Technical Design Report, CERN-LHCC-2013-021, LHCB-TDR-013, 2013.

[4] The LHCb Collaboration, Physics case for an LHCb Upgrade II - Opportunities in flavour physics, and beyond, in the HL-LHC era, LHCb-PUB-2018-005, CERN-LHCb-PUB-2018-005, 2018.

[5] J. Albrecht at al., Upgrade trigger \& reconstruction strategy: 2017 milestone, LHCb-PUB-2018-023,CERN-LHCb-PUB-2018-005, 2018.

[6] The CMS Collaboration,Techincal Proposal for a Timing Detector in the CMS experiment Phase 2 Upgrade, CERN-LHCC-2018-023, 2018.

[7] The ATLAS Collaboration, Technical Proposal: A High-Granularity Timing Detector for the ATLAS Phase-II Upgrade, CERN-LHCC-2018-023, LHCC-P-012, 2018.

[8] T. Behnke et al., ILC TDR Volume 4: Detectors, arXiv:1306.6329 [physics.ins-det]; Linssen, Lucie et al., Physics and Detectors at CLIC: CLIC Conceptual Design Report - arXiv:1202.5940 [physics.ins-det]; The CEPC Study Group, CEPC Conceptual Design Report: Volume 2 - Physics \& Detector, arXiv:1811.10545 [hep-ex]; FCC CDR, https://fcc-cdr.web.cern.ch.

[9] Y. Kudenko, Neutrino detectors for oscillation experiments, arXiv:1705.06059v1.

[10] S. Parker, A proposed vlsi pixel device for particle detection, Nucl. Instr. and Meth. A, 275(3), 494-516, 1989.

[11] M. Garcia-Sciveres and N. Wermes, A review of advances in pixel detectors for experiments with high rate and radiation, arXiv:1705.10150v3, 2017, and references within.

[12] H. F. W. Sadrozinski, A. Seiden, 4D tracking with ultra-fast silicon detectors, Rept. Prog. Phys. 81 (2018) no.2, 026101 (2017-12-18), DOI: 10.1088/1361-6633/aa94d3, e-Print: arXiv:1704.08666 [physics.ins-det] and references within.

[13] F. Simon, Silicon Photomultipliers in Particle and Nuclear Physics, arXiv:1811.03877v1, submitted to NIM A, 2018, and references within. 